\title{
Racing to Zipf's Law: \\ Race and Metro Population Size 1910-2010
}

\author{
Ricardo T. Fernholz* \\ Claremont McKenna College
}

\author{
Rory $\mathrm{Kramer}^{\dagger}$ \\ Villanova University
}

February 16, 2021

\begin{abstract}
Residential segregation scholarship traditionally focuses on segregation within metropolitan areas. Concurrently, urban economists have identified that cities and metro areas within a coherent, connected system of cities have population distributions that follow a power law. Further, subpopulations with equal mobility should also have population distributions that follow a power law. Using this insight, we introduce a novel method for identifying whether or not racial segregation between metro areas is due to geographical patterns of immigration into coherent systems or due to constrained mobility. We demonstrate the potential of this method by measuring the coherence of the U.S. urban system from 1910-2010 for the white, Black, Asian, and Hispanic populations. Black residents were only distributed across cities in a coherent system after the Great Migration, while Asian and Hispanic residents reached coherence more quickly and stayed coherent more consistently. It appears the Black reverse migration to the south has unsettled their power law distribution. We also find that the size of those networks differ across populations, and these differences are not an artifact of the relative size of the different populations. We conclude with more potential applications of the methodology.
\end{abstract}

Keywords: Zipf's law, power laws, race, mobility, random growth

${ }^{*}$ Claremont McKenna College, 500 E. Ninth St., Claremont, CA 91711, rfernholz@cmc.edu †Villanova University, 800 E. Lancaster Ave, Villanova, PA 19085, rory.kramer@villanova.edu 


\section{Introduction}

Scholarship on racial residential segregation has traditionally focused on urban populations, in part because of data and methodological benefits and in part due to the argument that the rise of racial segregation mirrored the migration of Black Americans from rural southern areas into northern cities (Massey and Denton, 1993). Using metrics such as the dissimilarity index, centralization index, and isolation index, this scholarship demonstrated that the U.S. first saw "hypersegregation" in the second half of the 20th century and that segregation was at its worst in northern rustbelt cities (Charles, 2003).

More recent scholarship has expanded the study of racial segregation by rethinking the scale of segregation, the history of segregation, and the location of interest. First, GIS has allowed scholars to shift from using administratively defined proxies of neighborhoods to egocentric or spatially adjusted neighborhood concepts to better appreciate the scale of segregation (Wong, 1993; Lee et al., 2008). The experience, for example, of living in a predominantly Black neighborhood that is adjacent to a predominantly white neighborhood or to a racially mixed neighborhood may be socially distinct from living in a predominantly Black neighborhood that is surrounded by other predominantly Black neighborhoods.

A second line of scholarship uses recently released census microdata from the first half of the 20th century to argue that the prior segregation literature underestimated the extent of segregation in the Jim Crow south because of its reliance on aggregated data and administratively defined neighborhood proxies (Logan and Parman, 2017; Logan and Martinez, 2018; Shertzer and Walsh, 2019). This literature also finds that segregation in the Jim Crow south developed earlier than previously thought. While this research does not invalidate the claim that northern industrial segregation was particularly extreme and problematic, it challenges the claim that such segregation was created as a replacement to Jim Crow style social control and instead suggests that it was originated alongside Jim Crow before expanding rapidly in the north.

Finally, a third field of research has followed the rise, fall, and revitalization of the urban core by studying segregation outside of major, hypersegregated cities (Lichter et al., 2015; Parisi et al., 2017). Urban segregation was, as long noted, in large part accelerated by the creation and subsidization of suburban development as a white space (Jackson, 1987; Rothstein, 2017). Yet the suburbs, today, are often sites of increasing diversification, by both international immigration and internal migration out of the city (Lacy, 2016). At the 
same time, suburbs have experienced an increase in poverty and, as geographies created for an idealized white middle class, are often ill-equipped to provide necessary social services for poor residents (Kneebone and Berube, 2013; Allard and Allard, 2017).

Across all of these subfields, isolating a specific urban or metro area to measure internal levels of segregation has been taken as a methodological given. But the isolation of individual metro areas from each other is an unstudied assumption. Some social network research has examined this issue by following individual migration patterns (South and Crowder, 1998; Crowder et al., 2012), but part of the difficulty is a lack of a measure for crossmetro segregation. Using racial population distributions across metro areas can demonstrate that some areas are disproportionately white, Asian, Hispanic, and/or Black, but historical patterns of racialized international migration or enslavement likely explain many of those differences. In fact, scholarship on Black internal migration indicates that the historical connection to southern spaces has led to a return migration for some Black families out of the more heavily segregated north (Pendergrass, 2013; Robinson, 2014).

To date, we know of no measures of national level patterns of segregation that are not, at their core, measures of individual metro segregation patterns. If properly constructed, such measures could help to understand whether or not racial groups internally migrate with equal freedom across metro areas even as they may choose different metro areas due to different racialized histories of migration. In the next section, we introduce the use of power laws and Zipf's law for the distribution of metro area populations in an urban system and apply these methods to subpopulations as a novel means of measuring the relative mobility of different groups across metro areas.

\section{Power Laws, Zipf's Law, and City Size Distributions}

A power law, or Pareto distribution, in the city size distribution refers to a log-linear relationship between the population size of each city and its rank. Thus, if a city with population $s_{i}$ has population size rank $k$ given by

$$
\log k=\beta_{0}+\beta_{1} \log s_{i},
$$


where $\beta_{0}$ and $\beta_{1}<0$ are constants, then the city size distribution follows a power law. If $\beta_{1}=-1$, then the city size distribution satisfies Zipf's law. Note that (1) implies that the slope of the log-log plot of city size versus rank will be a straight line with slope $\beta_{1}<0$. Following much of the literature, we use the terms power law and Zipf's law interchangeably since we are not concerned with the particular slope of the log-log plot of size versus rank, as long as that slope is not too far from minus one.

Soo (2005) documents that city size distributions in many different countries follow power laws of the form (1) with a constant $\beta_{1}$ approximately equal to minus one. Dittmar (2011) shows that historical metro area populations in Europe follow a power law after 1500 once labor and capital mobility had risen and locational fundamentals had become less important for city growth. Furthermore, scholars note that Zipf's law holds most strongly when an urban system (e.g., a province or nation) is coherent and co-evolving (Arshad et al., 2018). Research in this field has demonstrated that power laws hold in contemporary U.S. census "places" (Eeckhout, 2004), "natural cities" (Jiang and Jia, 2011), and metropolitan areas (Krugman, 1996; Gabaix, 1999). In sum, if city populations follow power laws, this is evidence those cities constitute an urban system.

Conceptually, Zipf's law should apply for subpopulations of cities if those subpopulations are randomly selected (Fernholz and Fernholz, 2020). For example, if a power law holds for the whole metro population, it should hold for the male population and female population separately, as the two do not segregate residentially. Similarly, if a power law holds for American metro areas and racial groups have equal mobility among those metro areas, then power laws should hold for each individual racial group as well. Crucially, Dittmar (2011) demonstrates that a power law only holds in Europe once areas reach a certain level of urbanization and coherence. We extend that insight by arguing that if subpopulations have similar or distinct city size distributions then this determines whether those subpopulations have similar or different levels of urbanization and coherence.

Importantly, the specific rank order of cities is irrelevant to this application of Zipf's law to subpopulations. In other words, that a given city may rank high for one subpopulation and low for another will not affect this measure, as long as the distribution of that population across metro areas follows a power law. To use one metro area as an example, while the San Jose metro area is the 35th largest in the U.S. for total population in the 2010 census, it had the fourth largest Asian population of all metro areas in 2010 and $33 \%$ of its population was 
Asian. This could be indicative of the continued migration from Asian countries to traditional West Coast receiving areas, the influence of anti-Asian or anti-immigrant sentiment elsewhere in the U.S., or a combination of the two (Lee and Kye, 2016). Alternatively, we could read San Jose as particularly anti-Black, given its MSA is only $2 \%$ Black. Regardless of the specific history of San Jose, research methods currently do not have an adequate measure of whether or not segregation extends between metro areas. Historically, the U.S. Black population did not have full access to internal migration opportunities until well into the 20th century due to white efforts to restrict Black mobility via vagrancy laws and extralegal violence such as lynchings (Anderson, 2016; Shertzer and Walsh, 2019). We argue that we can test this proposition not only by examining specific local histories of inclusion or exclusion, but also by seeing whether or not the Asian or Black populations, for example, adhere to Zipf's law across metro areas to the same extent as other populations in the urban system.

\section{Theory}

Suppose that there are $i=1, \ldots, N$ cities, and that the size of each city $s_{i}$ evolves according to

$$
\log s_{i}(t+1)-\log s_{i}(t)=\mu+\sigma B_{i}(t)
$$

where $\sigma>0$ and each $B_{i}$ is independent and normally distributed with mean zero and variance one. The models of Gabaix (1999), Rossi-Hansberg and Wright (2007), and Dittmar (2011) all generate city size dynamics like (2) for modern economies in which there are no frictions that restrict labor or capital mobility. In this case, both the average and variance of city growth rates are equal to each other and do not depend on size or any other distinguishing factor. In order for this setup to yield a stationary city size distribution, however, it is necessary to introduce a minimum value for city size or a city birth/death process, and to set $\mu<0$ (Gabaix, 2009).

Let $s_{(k)}(t)$ denote the size of the $k$-th largest city at time $t$, so that

$$
s_{(1)}(t) \geq s_{(2)}(t) \geq \cdots \geq s_{(N)}(t)
$$

for all $t$. We use the parameters $k=1, \ldots, N$ to denote the rank of cities. Let and $s_{(k)}^{*}$ 
denote the size of the $k$-th largest city in the stationary city size distribution. Fernholz (2017) shows that the model (2) yields a stationary city size distribution that follows

$$
E\left[\log s_{(k)}^{*}-\log s_{(k+1)}^{*}\right] \approx-\alpha(\log (k)-\log (k+1))
$$

where $\alpha>0$ is a constant and the approximation improves as $k$ increases. Recall that a linear relationship between log city size and log rank as in (4) represents a power law. Fernholz and Fernholz (2020) extend this result in two directions. First, they explain that (4) also describes the stationary city size distribution for any subpopulation with city growth dynamics as in (2). Thus, any subgroup of the full population with similar behavior should also follow the same city size distribution as the full population. Second, Fernholz and Fernholz (2020) show that as the number of ranked cities $k$ grows to infinity, the parameter $\alpha$, which measures the slope of the log-log plot of city size versus rank, converges to one.

A natural extension of (2) posits that city growth rates and variances may vary depending on the size of a city. This is equivalent to a model in which the size of each city $s_{i}$ evolves according to

$$
\log s_{i}(t+1)-\log s_{i}(t)=\mu_{r_{i}(t)}+\sigma_{r_{i}(t)} B_{i}(t)
$$

where $r_{i}(t)=k$ if city $i$ is the $k$-th largest city at time $t$. Note that this definition implies that $r_{i}(t)$ denotes the size-rank of city $i$ at time $t$. The rank-based variation in growth rates and volatilities in (5) captures the real-world possibility that labor and capital mobility are imperfect, and that individuals may not be able to costlessly move themselves or their capital to the most desirable cities. In such cases, we might expect that the growth rates of the largest cities are on average below those of smaller cities. Systematically, then, this argues that constraints on labor mobility would disproportionately affect the growth rate of larger cities below their expected size given a power law distribution. The same would be true of subpopulations.

Fernholz (2017) shows that the model (2) yields a stationary city size distribution provided that the rank-based growth rate parameters $\alpha_{1}, \ldots, \alpha_{N}$ satisfy a regularity condition such that higher-ranked, larger cities do not on average grow faster than lower-ranked, smaller cities. In such cases, the city size distribution no longer necessarily follows a power law in which the log-log plot of city size versus rank is linear. 
Proposition 3.1. Suppose that the rank-based growth rates $\mu_{1}, \ldots, \mu_{N}$ satisfy

$$
\mu_{1}<\mu_{2}<\cdots<\mu_{N}
$$

and the rank-based variance parameters satisfy $\sigma_{k}=\sigma>0$, for all $k=1, \ldots, N$. Then, the stationary city size distribution satisfies

$$
E\left[\log s_{(k)}^{*}-\log s_{(k+1)}^{*}\right] \approx-\alpha(k)(\log (k)-\log (k+1)),
$$

where $\alpha(k)>0$ is a strictly increasing function of rank $k$.

Proposition 3.1 implies that a version of the model (5) in which lower-ranked, smaller cities have higher average growth rates will have a stationary city size distribution in which the slope of the log-log plot of city size versus rank is steeper at lower ranks. Thus, if some factor restricts the growth of larger cities relative to smaller cities, then the city size distribution will deviate from a power law in such a way that large, higher-ranked cities will be smaller than a power law predicts. As before, this is true for the full population city size distribution as well as for subpopulation city size distributions. In other words, if mobility for a (sub)population is constrained across metro areas, then the pattern of deviation from a power law distribution should be that the largest cities have a smaller than expected population size.

\section{Data}

Data for this project come from decennial censuses. The U.S. Census Bureau first identified metropolitan areas for the 1950 census. For each census from 1950-2010, we use U.S. Census demarcations of metropolitan areas. For 1910, as a comparison before the Great Migration in the U.S. of Black citizens from the largely rural south into the urbanized north, we follow NHGIS protocol and use data at the county level that are then matched to 1950 metropolitan area definitions. Any error from including overly rural areas in 1910 in metro areas as defined by 1950 should bias our estimates toward finding Zipf's law applies for the Black population, as Black Americans disproportionately lived in rural areas in 1910. The data for 1970, 
1980, 1990, and 2010 were downloaded from the Social Explorer dataset at the Metro Area level. Data for 2000 were downloaded from the American Communities Project at Brown University at the metro area level.

\section{Empirical Evidence}

Figure 1 plots the log population of U.S. metro areas (as a fraction of the total) versus their log population-rank from 1910-2010. Recall that a linear relationship between log city size and log rank represents a power law, or Zipf's law. According to Figure 1, the U.S. metro size distribution has approximately followed Zipf's law throughout the last century, although the distribution curve did become more curved in 2010.

Figure 2 presents a similar log-log plot of metro population versus rank for 1910 and 1950, but considers the white and Black U.S. populations separately. According to the figure, the distribution of Black metro populations deviated substantially from Zipf's law in 1910, with only the very largest, top 20-30 ranked metros following a straight line in the plot. By 1950, however, the distribution of Black metro populations follows the straight line pattern of a power law much more closely, with almost all of the top 100 ranked metros generating a relatively linear log-log plot. In contrast, the distribution of white metro populations is close to a straight line at all ranks in both 1910 and 1950.

Figures 3 and 4 more closely examine this transition towards Zipf's law for U.S. Black metro populations during the twentieth century. According to Figure 3, the log-log plot of Black metro populations versus rank consistently grew more linear from 1910 to 1970, with the 1970 distribution following the straight line pattern of Zipf's law for more than 100 ranked metro areas. There is much less change in Figure 4, which shows the Black metro population distribution from 1980-2010. The different log-log plots in this figure are much closer together than those in Figure 3, which suggests that the transition of the Black metro size distribution that occurred in the early part of the twentieth century was largely finished by the later part of the century. Finally, Figure 5 shows the size distribution of U.S. Hispanic metro populations from 1970-2010. According to the figure, the distribution of Hispanic metro populations deviated most noticeably from a power law in 1970.

The deviation from a power law for Black metro populations shown in Figures 2 and 3 
closely matches with the prediction of Proposition 3.1. According to the proposition, if some factor constrains the growth of the largest cities within an urban system, then the resulting city size distribution will feature a log-log plot of city size versus rank in which the slope is less steep for top-ranked, larger metros. This exact pattern is observed for Black metro populations in 1910. In the context of Proposition 3.1, then, this specific deviation from a power law suggests that there existed substantial constraints on the ability of the Black population to freely move from one U.S. metro area to another in the first decades of the twentieth century. Such mobility constraints would likely dampen the growth rate of the largest, most desirable Black metro areas and hence lead to the curved size distribution of 1910 (Figures 2 and 3).

Dittmar (2011) introduces a method for quantifying the changes in size distributions that we observe in Figures 1-5. Let $s_{i}$ denote the population of city $i$ and $r_{i}$ denote the population rank of city $i$. Following Dittmar (2011), we estimate the regression

$$
\log r_{i}=\beta_{0}+\beta_{1} \log s_{i}+\epsilon_{i}
$$

using the non-parametric estimator based on the median developed by Theil (1950). For this estimator, the slope coefficient $\beta_{1}$ is calculated as the median of all slopes generated by pairs of data points. Correspondingly, the constant $\beta_{0}$ is calculated as the median over all data points $i$ of

$$
\log r_{i}-\hat{\beta}_{1} \log s_{i}
$$

where $\hat{\beta}_{1}$ is the median slope estimator of $\beta_{1}$.

The median regression estimator of Theil (1950) is designed specifically for applications in which outliers are potentially significant, and provides less biased estimates in such scenarios. It also performs well in small samples. Furthermore, the median estimator is competitive with OLS estimates of $\beta_{0}$ and $\beta_{1}$ in environments in which there are no major outliers (Dittmar, 2011). Given these properties and the substantial deviations from a power law that we observe in Figures 2 and 3, the median estimator of Theil (1950) is particularly well-suited for our application.

Table 1 reports the mean square deviation in percent between the actual metro size distributions and the estimated power law distribution (8) using the Theil median estimator for U.S. white, Black, Hispanic, and Asian populations from 1910-2010. In this table, the 
actual and estimated metro size distributions are constructed using only those metros with populations equal to at least $1 \%$ of the largest city population for each group. The table shows a dramatic decline in the deviation between the actual distribution and the estimated power law distribution for the Black population from 1910-1970. The mean square deviations from a power law for Black metro populations are similar to that for white metro populations from 1970 on, except for 2010 which we discuss below.

Adherence to Zipf's law as measured by Figures 1-5 and Table 1 demonstrates the coherence of an urban system for a given population. Confirming prior research, our analyses show the U.S. as a whole is a coherent urban system and that is also true for the white population who have historically had complete domestic labor mobility across all the years in our data. On the other hand, that coherency only became true for the Black population after the Great Migration, and did not reach parity with whites until the 1970 census. That coherence began to slip in 1990 for both Black and white Americans, as the mean square deviation grew for both groups. However, that growth was small and reversed for whites between 2000 and 2010 .

Conversely, such a reversal did not occur for Black urban system coherence, as the mean square deviation grew from 3.79 in 1990 to 5.09 in 2000 to 8.60 in 2010. Turning back to the figures, there is a small but noticeable decline in the population shares in the top two metros for Black residents. New York had the largest Black population of all metros for all three censuses, but its growth stalled between 1990 and 2000 and it lost roughly 100,000 Black residents between 2000 and 2010 while remaining the top population center. Nonetheless, New York's stalled growth and decline while the total Black population across our sample grew led to it being below expectations for a power law distribution. The second largest metro area was Chicago in 1990 and 2000 and saw the same pattern - slow growth between 1990 and 2000 and then a slight decline between 2000 and 2010. In fact, Chicago's decline was enough for it to be supplanted in the rankings in 2010 by the growing Atlanta MSA. Similar patterns exist for other Mid-Atlantic and Midwest metro areas and Atlanta is joined by Miami, Houston, and Dallas as fast growing MSAs with regard to their Black populations.

We hypothesize that the growing deviation from the median estimator from 1990-2010 is not driven by a loss of spatial mobility for Black residents, but rather a realignment of their distribution from northern postindustrial cities toward growing southern economies that is not yet complete and thus not yet fully coherent (Pendergrass, 2013; Robinson, 2014). 
Similar patterns of stagnation are also apparent amongst the white population in major northern and midwestern MSAs, but that stagnation is not matched by significant growth in southern MSA white populations and thus it did not lead to a similar growth in deviation from the median estimator for the white distribution. We note, however, that the deviation from a power law for Black city populations in 2000-2010 shown in Figure 4 is of smaller than expected top-ranked cities, the same pattern as observed for Black city populations in 1910 as shown in Figure 3 (although much less pronounced). Proposition 3.1 implies that such deviations from a power law indicate some form of constraint. We theorize the constraint in recent decades is about the growing crises of affordability for working class people in metros like New York and Chicago, and less so about an explicitly racialized lack of mobility such as occurred during the era of Jim Crow vagrancy laws and violence during the first half of the 20 th century.

Turning to Hispanic and Asian population distributions, by 1980 both were embedded in coherent urban systems. Because the total Asian population was relatively small in 1970 - only 1.5 million in the entire US, less than one percent of the total U.S. population, we did not measure the Asian distribution in 1970. Nonetheless, that a population that more than doubled in size between 1970 and 1980 — from 1.5 million to 3.7 million — fit a power law distribution well in 1980 indicates that they were either already established in a coherent urban system or quickly developed such a system. This may be related to the relative level of human capital many Asian migrants brought to the U.S. on top of a long history of Asian settlement in the American west that offered a base for labor integration. Similar to the Black population distribution, the Asian distribution saw an increase in its deviation from the median power law estimator between 2000 and 2010, from 2.82 to 5.16. While still relatively small, that growth is noticeable as it is the only time period in which the Asian population distribution deviated from the estimator by more than 3.05. The Hispanic population similarly had a long history of living in the American west and southwest, but in 1970 nonetheless appeared to not represent a coherent urban system, with a deviation of over 13. That deviation quickly declined by 1980. We caution that this finding may be due to a substantial undercount (by more than 5\%) of the Hispanic population in 1970 combined with a miscount of hundreds of thousands of non-Hispanic whites as Hispanic (Cohn, 2010). Unlike the Black and Asian populations, the first decade of the 21st century saw Hispanic population distributions become the closest to the median estimator (2.59) of all of our 
groups. Overall, however, all of the groups - excluding Black Americans — remain close to the median estimator and demonstrate consistent coherence since at least 1980 .

As mentioned earlier, we restricted the sample for each analysis to metro areas with at least $1 \%$ of the population of the largest metro area. For example, for our analysis of 2010 MSAs, this means that we only include MSAs that had a population of at least 188,971 (1\% of the population of New York, the largest MSA in 2010). In our analysis of 2010 white populations, each MSA had to have at least 92,338 white residents (again, 1\% of the population of the New York MSA). However, for Asians, that was 1\% of the Los Angeles metro area, or 20,517 Asian residents in a given MSA. Table 2 reports the number of metros included in the mean squared deviation calculations from Table 1. Thus, this table shows how many metro populations are equal to at least $1 \%$ of the largest city population for U.S. white, Black, Hispanic, and Asian populations from 1910-2010. In the appendix, we instead restrict results to metro areas with $0.1 \%$ of the largest population. In these analyses, the low population tail gives a clear curve to the log-log plot of size versus rank, indicating that the populations do not fit a power law distribution when including metro areas with small populations of a given racial group (see Figures 6-8 in the appendix). These curves were especially egregious in earlier years with smaller population thresholds. For example, the smallest Black population we used in 1950 was 10,134 Black residents per MSA. Were we to use the smaller cut-off, metro areas with as few as 1,135 Black residents would be included in our sample.

Table 2 shows a second component to the power law distribution of populations. Where Table 1 measures the coherency of the distribution, Table 2 measures the completeness of a distribution, or to return to Dittmar (2011)'s study of European cities, the relative level of urbanization. Take, for example, the total number of metro areas included for each racial group in 2010. For whites, nearly 300 metro areas are included out of a possible 371 . In fact, this is greater than the number included for the total population (224), demonstrating not only that white labor mobility is in a coherent urban system, but also that the urban system is broad across almost the entire U.S. metro map. On the other hand, while Black, Hispanic, and Asian metro samples all increased in number, they are far smaller than the white urban system, indicating that while each racial population exists in a coherent urban system, these coherent urban systems are not as complete as the white urban system across U.S. metro areas. This also explains why the white cut-off includes more metro areas than 
the total population cut-off, as the total population cut-off is based on the inclusion of both non-white and white populations into one urban system.

Nor is this an artifact of population size within the United States. The Hispanic population outnumbered the Black population in the 2010 census, yet their power law sample includes 44 fewer metro areas. In this case, that is driven by a much higher cut-off for Hispanic metros $(57,000)$ compared to Black metros $(31,700)$, thanks to the much higher concentration of Hispanic residents in the LA metro compared to Black residents in the New York metro.

In Tables 3 and 4 in the appendix, we report the mean squared deviation from the estimated power law distribution and the total number of metros using a lower population threshold ( $0.1 \%$ of that subpopulation's largest city size). Here, the smaller threshold means hundreds more metros are included in the distributions. Across all racial groups, and for the total population, the mean squared deviation grew, but the extent is substantially different. For whites, Asians, and the total population, the increase in the deviation is between one and three percentage points. However, for Black and Hispanic populations, the growth is substantial - never reaching below 23 percent for the Black population, and never reaching single digits for Hispanics. We note that the temporal pattern toward coherence is largely similar, though the large growth in deviation from a power law for Hispanics between 2000 and 2010 does not occur in our main, more conservative threshold results. Looking at graphs of the distributions, it is clear that the deviation is driven by the cities with very small populations of a given racial or ethnic group. Thus, we recommend using the $1 \%$ threshold in order to avoid abnormally high deviations driven by metro areas with very small populations.

\section{Discussion}

Scholars studying racial segregation have long appreciated the importance of scale to understanding residential segregation. Primarily, that appreciation has focused on problems of identifying the local neighborhood and of incorporating spatial dependence into measures of residential segregation (Barros and Feitosa, 2018; Dean et al., 2019; Kramer, 2017, 2018; Lee et al., 2008; Wong, 1993). We advance an alternative methodological appreciation for 
the scale of residential segregation — instead of focusing on the level of segregation between neighborhoods within a metro area, we focus on segregation across metro areas. Expanding on scholarship that shows that metro areas in a coherent urban system follow power law population distributions, we argue that the same should be true of subpopulations of those metro areas, if those subpopulations have the same mobility as other area residents. Next, we apply that insight to racial populations in the U.S. and explore the level of urban system coherence and the size of that system for racial and ethnic groups within the U.S. Here, we discuss the implications of our findings that each of the major racial/ethnic groups in the U.S. generally do demonstrate a coherent urban system after 1970, though the sizes and scopes of those systems vary, and focus on other potential applications of using Zipf's Law as a test of urban system coherence.

As with other measures of residential segregation, this method for analyzing city size distributions for subpopulations can be used to study any type of subpopulation, not only racial/ethnic groups. For example, scholars interested in following the possible rise of the "creative class" in cities could use this method to determine whether or not creative class jobs are in a coherent urban system, or if they are overly represented in a few "winner" cities. If distributions show divergence from a power law distribution, that would identify not only topics of interest for understanding what is and is not part of a coherent urban system, but also where a particular population is over or underrepresented compared to expectation.

Not only does our analysis demonstrate whether or not a coherent system exists, we also argue that the distribution shows the extent of that coherent system. In our comparison of results from Tables 1 and 2 and the appendix, we show that once we reach a small enough population, the urban system loses coherence for Black and Hispanic subpopulations. Alternatively, this may instead be evidence of limited mobility for Black and Hispanic residents, especially given the smaller difference between the mean squared deviation for Asians using the $1 \%$ and $0.1 \%$ population thresholds, who represent the smallest subpopulation in our tables. Thus, comparing relative adherence to a power law for populations offers not only a metric of coherence of a system but also the completeness of coverage of that system across metro areas.

We note also that the 21st century saw a turn away from coherence for Black and, to a lesser extent, Asian populations. We argue that this is evidence of the stagnation of the Black population in large northern and midwestern cities and the reverse migration to 
growing southern metro areas, though we note that similar stagnation did not show the same impact on white population distributions. For Asians, we see a similar stagnation, as in 2010, for the first time, the top two ranked cities have nearly identical Asian populations (difference of just over 50,000 Asian residents), whereas in prior years, Los Angeles had hundreds of thousands more Asian residents than New York. However, unlike the stagnation reported for other racial groups, this is a difference in two steadily growing populations, as Los Angeles saw 400,000 more Asian residents in 2010 compared to 2000 while New York saw a growth of over 550,000. If those trends continued between 2010 and 2020, New York would surpass Los Angeles and the gap between the two would grow and the Asian city size distribution would move closer to the median estimator.

While we demonstrate that adherence to Zipf's law for subpopulations can identify the extent and coherence of an urban system for subpopulations on top of prior arguments showing that Zipf's law applies for total populations of cities or metro areas, we do note some limitations. First, deviation from Zipf's law is not inherently evidence of incoherence of an urban system. The above paragraph hypothesizes that the growing deviation in the 21st century was not because the urban system of Black Americans was growing less coherent, but rather that a snapshot of a gradual transition from one system to a new one will appear to be incoherent. This is no different from concerns for other measures of segregation in which single time measures of areas undergoing gentrification or racial turnover can, depending on how far into the process it is, appear to be evidence of integration (Bader and Warkentien, 2016). Second, further work should determine exactly what threshold should qualify as deviating too far from a power law for coherence and how to determine the correct threshold for relative size for inclusion into the distribution. As the appendix demonstrates, the lower end of the distribution can radically alter a distribution that otherwise fits a power law well. Instead of determining specific thresholds as arbitrary cutoffs, we chose to and encourage future research to explore multiple thresholds and instead focus on comparisons across times or populations rather than comparisons to pre-determined limits.

Before the Civil Rights Movement, whites limited Black mobility through legal and extralegal methods (Anderson, 2016). While legal efforts to enforce segregation and limit Black mobility were reversed in the 1960s, scholarship on segregation within metro areas provides overwhelming evidence that such efforts did not ameliorate segregation and, if anything, segregation grew after the Civil Rights Movement (Charles, 2003). In this paper, we shift 
further outward, to study segregation across metro areas instead of within them. Here, we find that the Great Migration was not just a movement north by Black Americans, but a movement into a coherent urban system, largely catching up with the white American urban systems in coherence, if not in scope. For Asians and Hispanics, we find that coherence came quickly after the immigration law reform in 1965. However, in the 21st century, there is evidence that the reverse great migration and some changes in Asian residential geography are shifting those coherent urban systems. By recognizing that a co-evolving urban system should also co-evolve for subpopulations if all residents are equally able to migrate, scholars can not only demonstrate that city size distributions adhere to Zipf's law, but use that to test for whom and to what extent an urban system is coherent and co-evolving.

\section{References}

Allard, S. W. and S. Allard (2017). Places in need: The changing geography of poverty. Russell Sage Foundation.

Anderson, C. (2016). White rage: The unspoken truth of our racial divide. Bloomsbury Publishing USA.

Arshad, S., S. Hu, and B. N. Ashraf (2018, February). Zipf's law and city size distribution: A survey of the literature and future research agenda. Physica A: Statistical Mechanics and Its Applications 492, 75-92.

Bader, M. D. and S. Warkentien (2016). The fragmented evolution of racial integration since the civil rights movement. Sociological Science 3, 135-166.

Barros, J. and F. F. Feitosa (2018). Uneven geographies: Exploring the sensitivity of spatial indices of residential segregation. Environment and Planning B: Urban Analytics and City Science 45(6), 1073-1089.

Charles, C. Z. (2003, August). The dynamics of racial residential segregation. Annual Review of Sociology 29(1), 173-204.

Cohn, D. (2010). Census history: counting hispanics. Pew Research Center. 
Crowder, K., J. Pais, and S. J. South (2012). Neighborhood diversity, metropolitan constraints, and household migration. American sociological review 77(3), 325-353.

Dean, N., G. Dong, A. Piekut, and G. Pryce (2019). Frontiers in residential segregation: Understanding neighbourhood boundaries and their impacts. Tijdschrift voor economische en sociale geografie 110(3), 271-288.

Dittmar, J. (2011, August). Cities, markets, and growth: The emergence of zipf's law. mimeo, LSE.

Eeckhout, J. (2004). Gibrat's law for (all) cities. American Economic Review 94(5), 1429 1451.

Fernholz, R. T. (2017). Nonparametric methods and local-time-based estimation for dynamic power law distributions. Journal of Applied Econometrics 32(7), 1244-1260.

Fernholz, R. T. and R. Fernholz (2020, December). Zipf's law for atlas models. Journal of Applied Probability 57(4), 1276-1297.

Gabaix, X. (1999, August). Zipf's law for cities: An explanation. Quarterly Journal of Economics 114(3), 739-767.

Gabaix, X. (2009, 05). Power laws in economics and finance. Annual Review of Economics 1(1), 255-294.

Jackson, K. T. (1987). Crabgrass frontier: The suburbanization of the United States. Oxford University Press.

Jiang, B. and T. Jia (2011). Zipf's law for all the natural cities in the united states: A geospatial perspective. International Journal of Geographical Information Science 25(8), 1269-1281.

Kneebone, E. and A. Berube (2013). Confronting suburban poverty in America. Brookings Institution Press.

Kramer, R. (2017). Defensible spaces in philadelphia: Exploring neighborhood boundaries through spatial analysis. RSF: The Russell Sage Foundation Journal of the Social Sciences 3(2), 81-101. 
Kramer, R. (2018). Testing the role of barriers in shaping segregation profiles: The importance of visualizing the local neighborhood. Environment and Planning B: Urban Analytics and City Science 45(6), 1106-1121.

Krugman, P. (1996, April). Urban concentration: The role of increasing returns and transport costs. International Regional Science Review 19(1-2), 5-30.

Lacy, K. (2016). The new sociology of suburbs: A research agenda for analysis of emerging trends. Annual Review of Sociology 42, 369-384.

Lee, B., S. Reardon, G. Firebaugh, C. Farrell, S. Matthews, and D. O'Sullivan (2008, October). Beyond the census tract: Patterns and determinants of racial segregation at multiple geographic scales. American Sociological Review 73(5), 766-791.

Lee, J. C. and S. Kye (2016). Racialized assimilation of asian americans. Annual Review of Sociology 42, 253-273.

Lichter, D., D. Parisi, and M. Taquino (2015, August). Toward a new macro-segregation? decomposing segregation within and between metropolitan cities and suburbs. American Sociological Review 80(4), 843-873.

Logan, J. and M. Martinez (2018, January). The spatial scale and spatial configuration of residential settlement: Measuing segregration in the postbellum south. American Journal of Sociology 123(4), 1161-1203.

Logan, T. and J. Parman (2017, March). The national rise in residential segregation. The Journal of Economic History 7r(1), 127-170.

Massey, D. and N. Denton (1993). American Apartheid, Segregation, and the Making of the Underclass. Cambridge, MA: Harvard University Press.

Parisi, D., D. Lichter, and M. Taquino (2017, Jun). Remaking metropolitan america? residential mobility and racial integration in the suburbs. Socius 5, 1-18.

Pendergrass, S. (2013). Routing black migration to the urban us south: social class and sources of social capital in the destination selection process. Journal of Ethnic and Migration Studies 39(9), 1441-1459.

Robinson, Z. F. (2014). This Ain't Chicago: Race, Class, and Regional Identity in the Post-Soul South. UNC Press Books. 
Rossi-Hansberg, E. and M. L. J. Wright (2007, April). Urban structure and growth. Review of Economic Studies 74(2), 597-624.

Rothstein, R. (2017). The color of law: A forgotten history of how our government segregated America. Liveright Publishing.

Shertzer, A. and R. Walsh (2019, July). Racial sorting and the emergence of segregation in american cities. Review of Economics and Statistics 101(3), 415-427.

Soo, K. T. (2005, May). Zipf's law for cities: A cross-country investigation. Regional Science and Urban Economics 35(3), 239-263.

South, S. J. and K. D. Crowder (1998). Leaving the 'hood: Residential mobility between black, white, and integrated neighborhoods. American sociological review, 17-26.

Theil, H. (1950). A rank-invariant method of linear and polynomial regression analysis. Proceedings of the Royal Netherlands Academy of Sciences 53, 386-392.

Wong, D. W. (1993). Spatial indices of segregation. Urban studies 30(3), 559-572. 


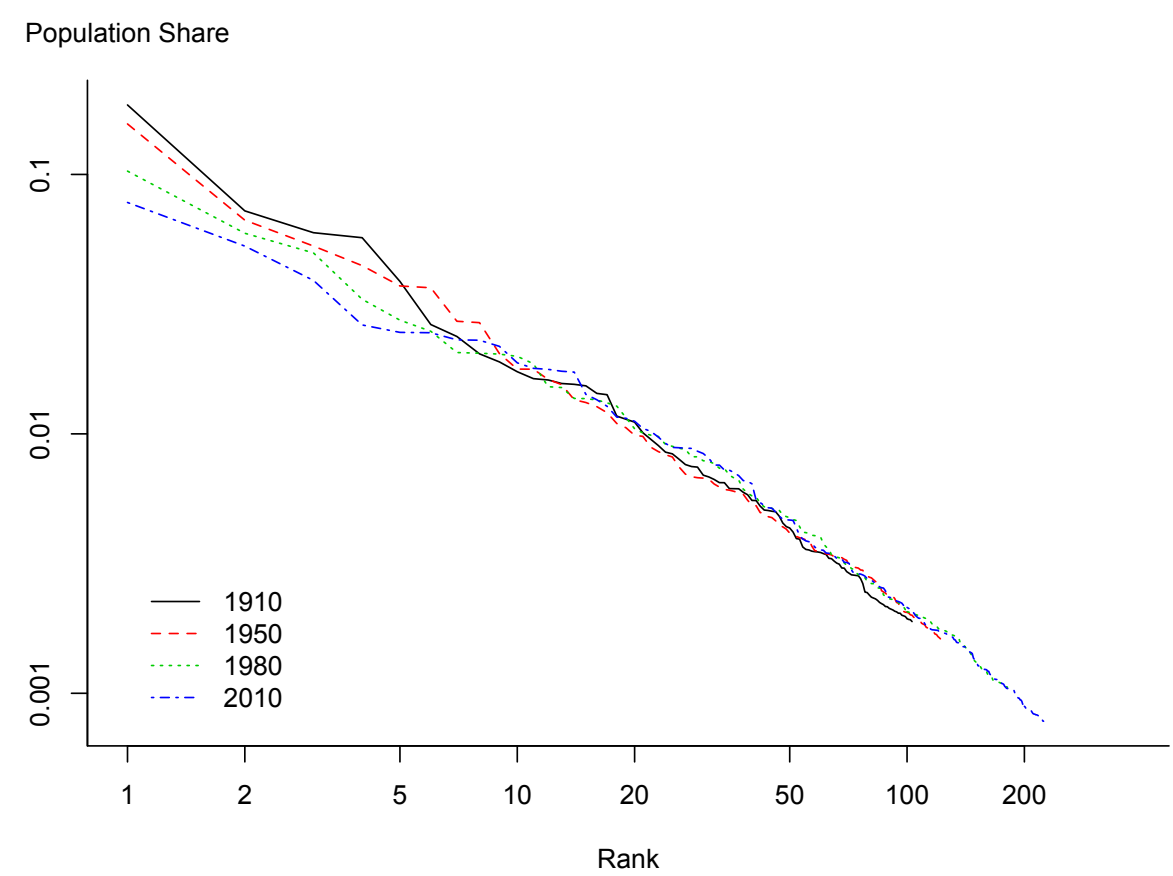

Figure 1: U.S. city size distribution, 1910-2010.

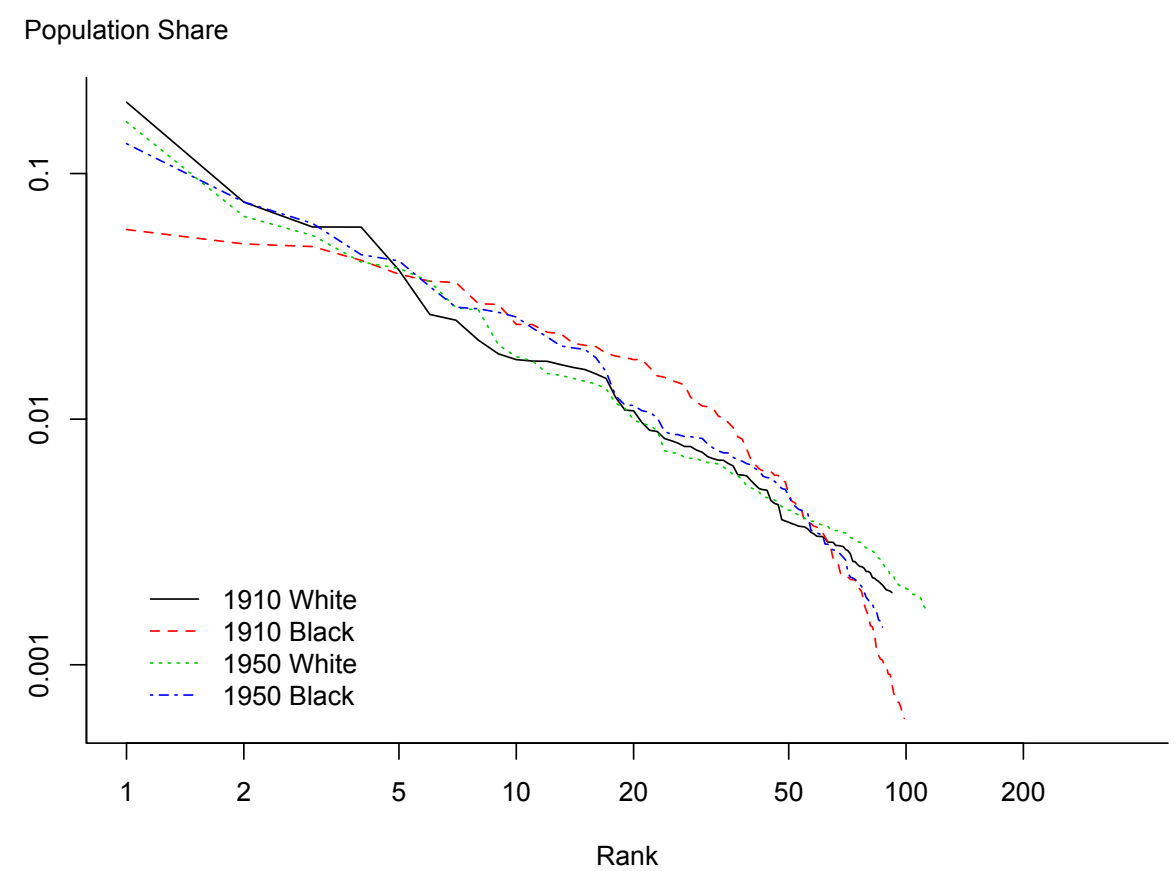

Figure 2: U.S. white and Black city size distributions, 1910-1950. 


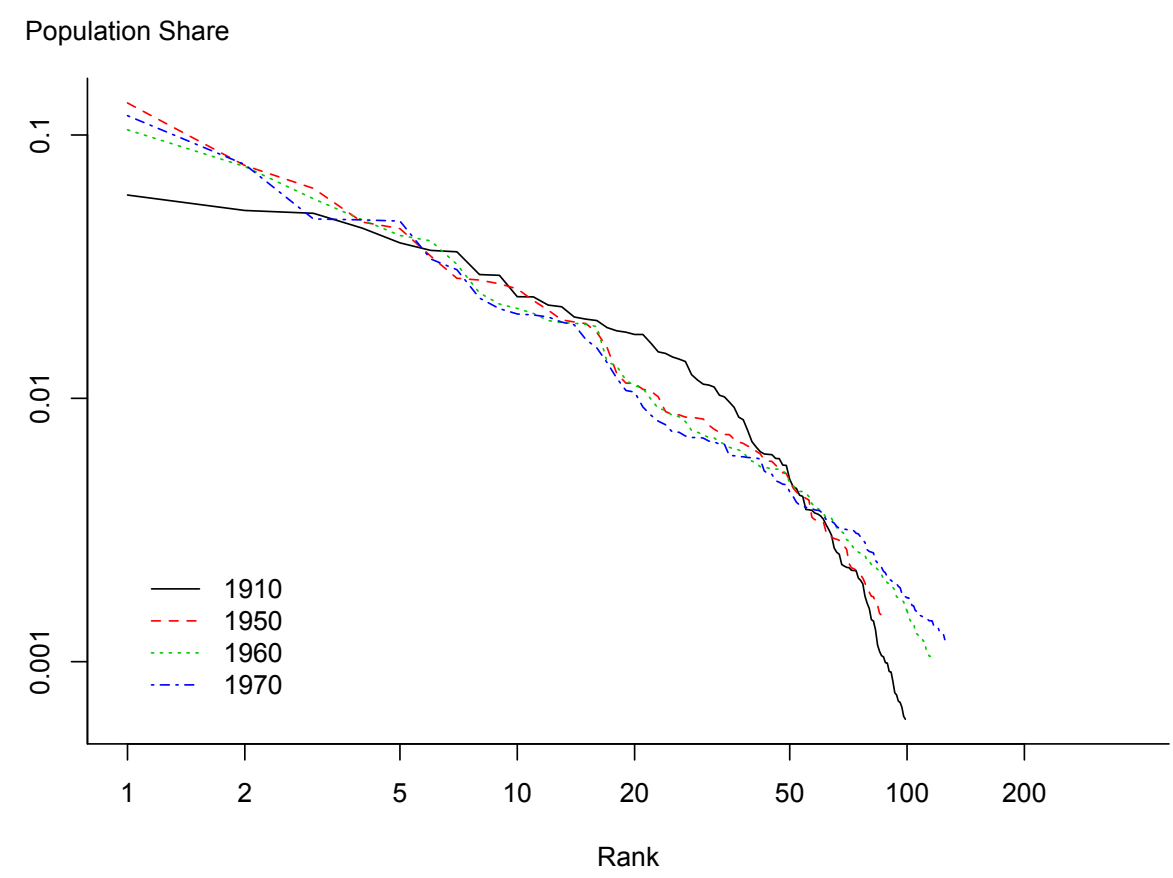

Figure 3: U.S. Black city size distribution, 1910-1970.

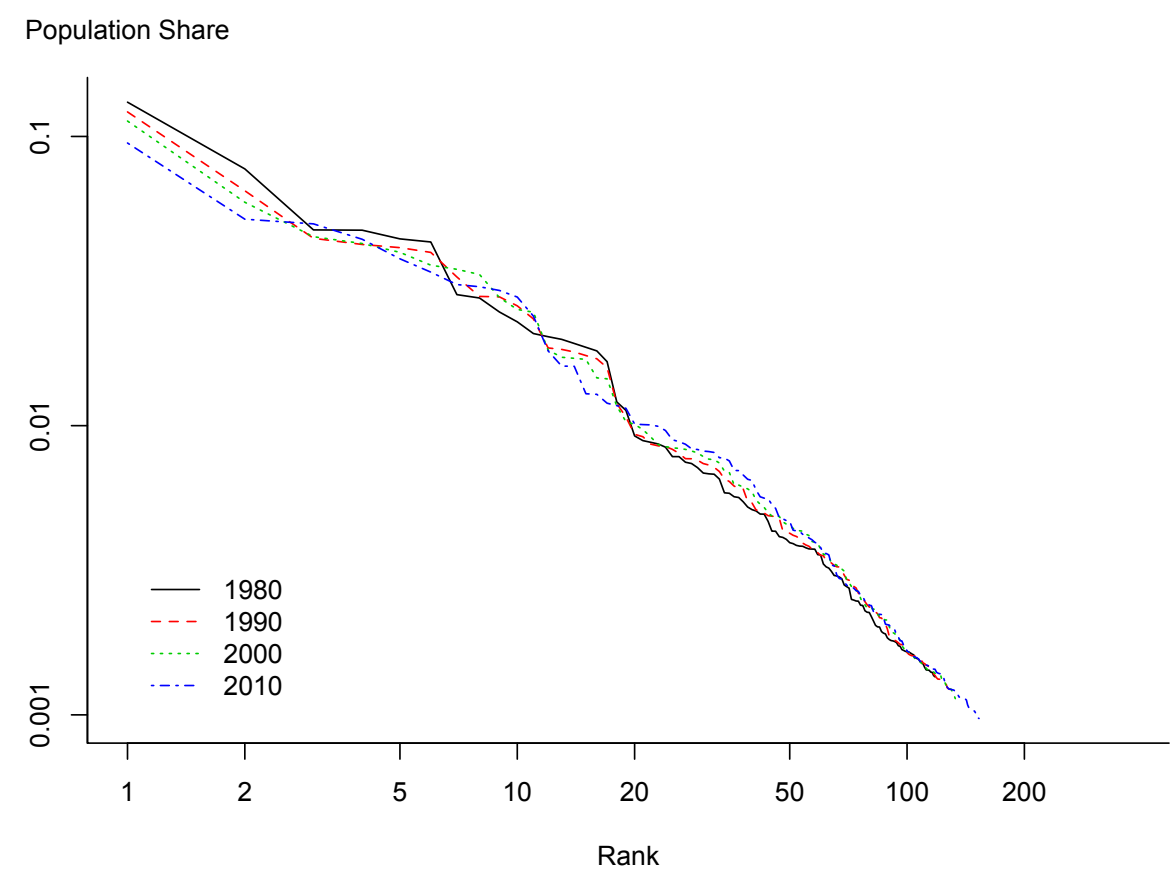

Figure 4: U.S. Black city size distribution, 1980-2010. 


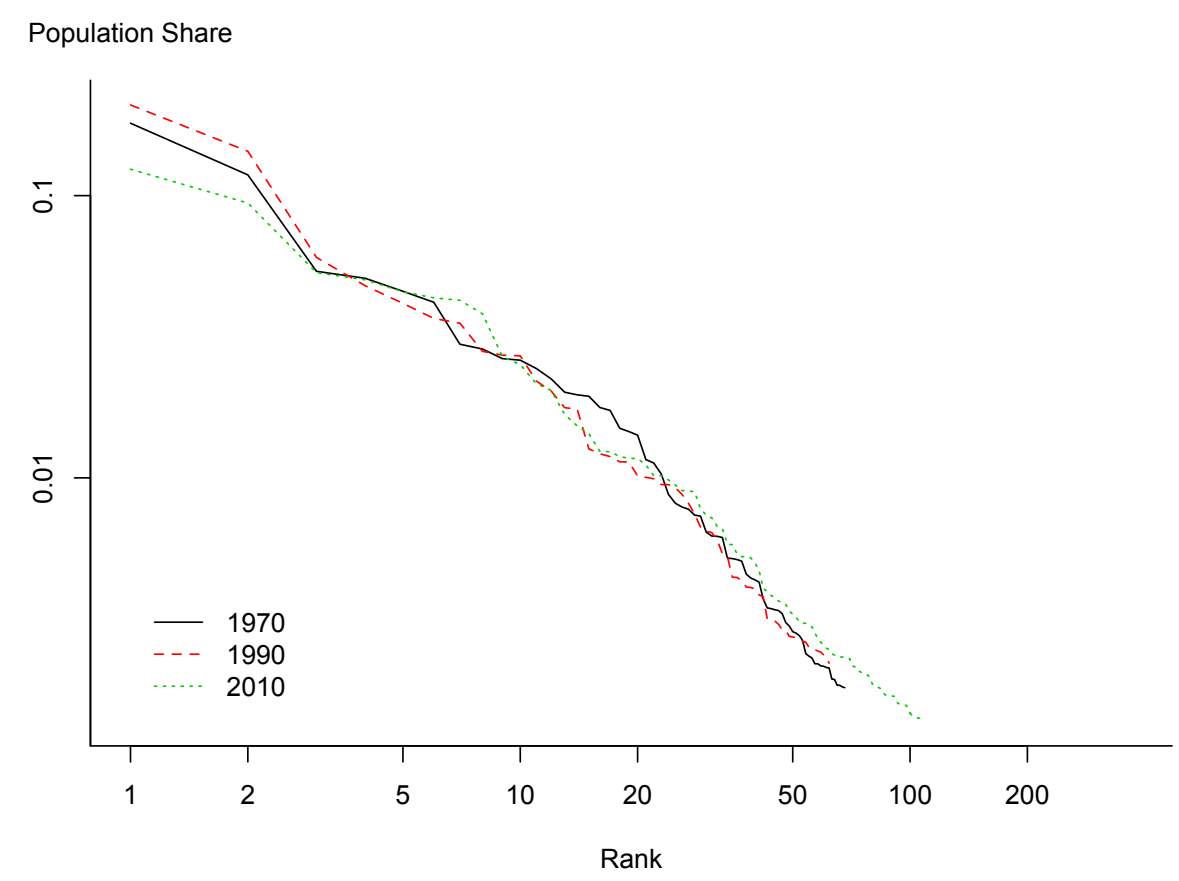

Figure 5: U.S. Hispanic city size distribution, 1970-2010.

\begin{tabular}{|c||c|c|c|c|c|}
\hline & All & White & Black & Hispanic & Asian \\
\hline 1910 & 1.07 & 1.31 & 62.08 & & \\
1950 & 0.43 & 0.57 & 7.01 & & \\
1960 & 2.90 & 3.50 & 7.47 & & \\
1970 & 5.22 & 4.34 & 3.18 & 13.15 & \\
1980 & 2.80 & 2.86 & 2.62 & 5.55 & 3.04 \\
1990 & 3.05 & 3.44 & 3.79 & 3.21 & 2.53 \\
2000 & 3.15 & 4.20 & 5.09 & 4.78 & 2.82 \\
2010 & 4.22 & 5.25 & 8.60 & 2.59 & 5.16 \\
\hline
\end{tabular}

Table 1: Mean squared deviation (\%) from estimated power law distribution for U.S. white, Black, Hispanic, and Asian populations, 1910-2010. 


\begin{tabular}{|c||c|c|c|c|c|}
\hline & All & White & Black & Hispanic & Asian \\
\hline 1910 & 103 & 92 & 99 & & \\
1950 & 126 & 112 & 87 & & \\
1960 & 180 & 181 & 115 & & \\
1970 & 211 & 218 & 125 & 68 & \\
1980 & 181 & 225 & 118 & 62 & 53 \\
1990 & 197 & 261 & 129 & 62 & 56 \\
2000 & 204 & 276 & 133 & 75 & 71 \\
2010 & 224 & 296 & 153 & 109 & 82 \\
\hline
\end{tabular}

Table 2: Number of cities with populations greater than or equal to $1 \%$ of largest city population for U.S. white, Black, Hispanic, and Asian populations, 1910-2010.

\section{Appendix}

In this appendix, we recreate Figures 1, 2, and 5 using using those cities with populations equal to at least $0.1 \%$ of the largest city population for U.S. white, Black, Hispanic, and Asian populations. Figure 6 shows a steep decline in population shares for the smallest cities in all years, which is clearly inconsistent with the linear relationship between log size and log rank of a power law distribution. The contrast between the approximately straight lines in Figure 1 and the sharp bends in the lines in Figure 6 is striking. We find a similar pattern in Figures 7 and 8, with a clear contrast between the curves in these figures at lower ranks and the approximately linear relationships of Figures 2 and 5.

We also recreate Tables 1 and 2 using the lower population threshold of $0.1 \%$. Table 3 shows that the mean squared deviations from estimated power law distributions for U.S. white, Black, Hispanic, and Asian populations using this lower threshold are much larger than the deviations reported in Table 1 using the $1 \%$ population threshold. This result is consistent with the highly non-linear relationships between log population share and log rank shown in Figures 6-8. 


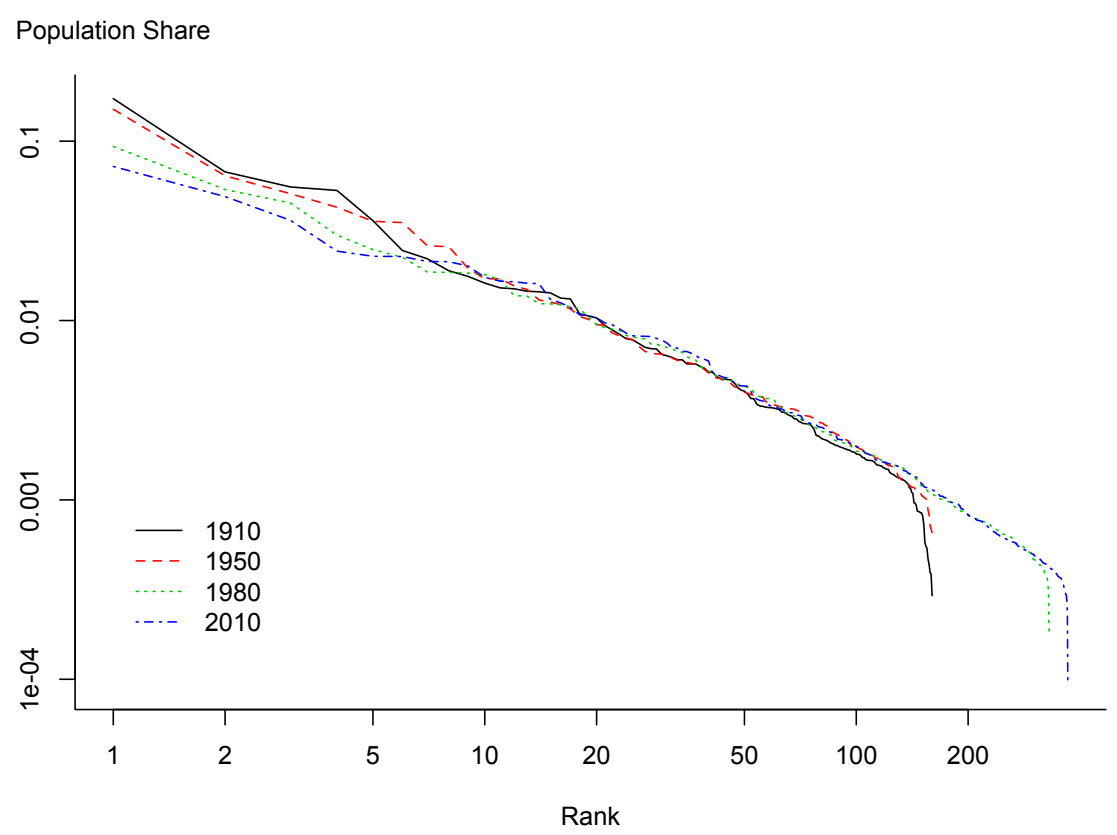

Figure 6: U.S. city size distribution using all cities with populations equal to at least $0.1 \%$ of the largest city population, 1910-2010.

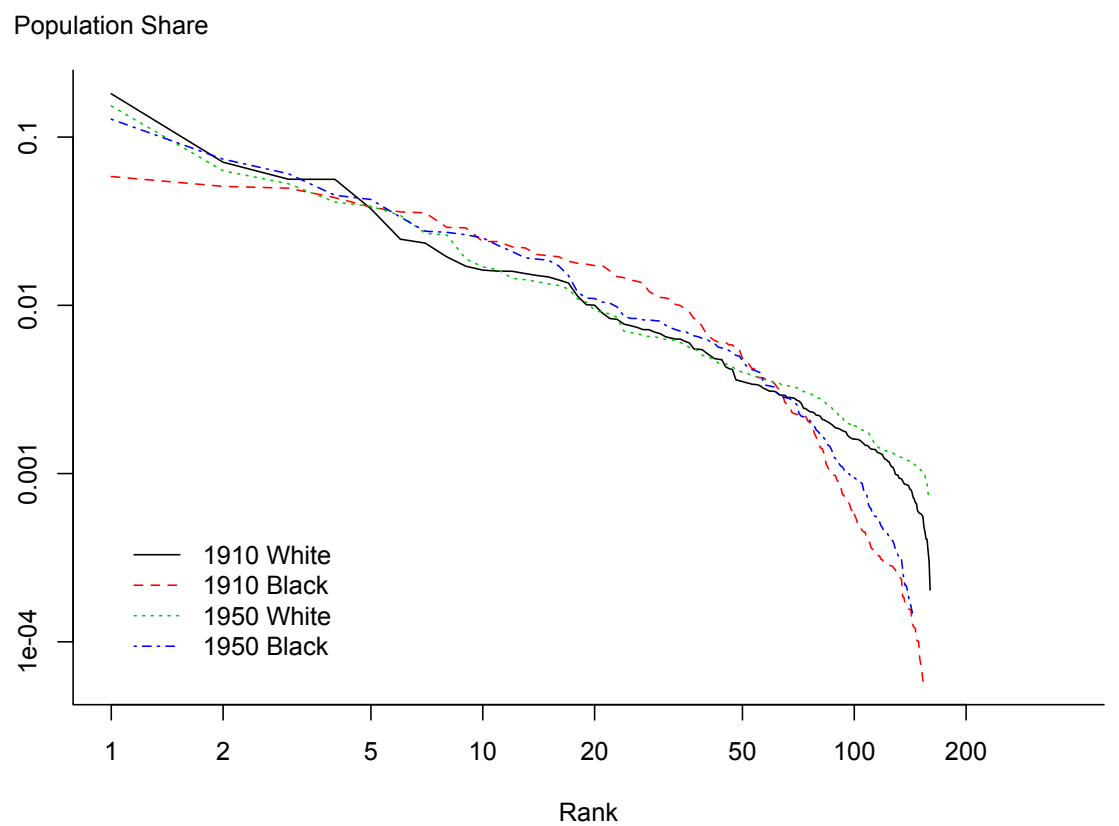

Figure 7: U.S. white and Black city size distributions using all cities with populations equal to at least $0.1 \%$ of the largest city population, 1910-1950. 


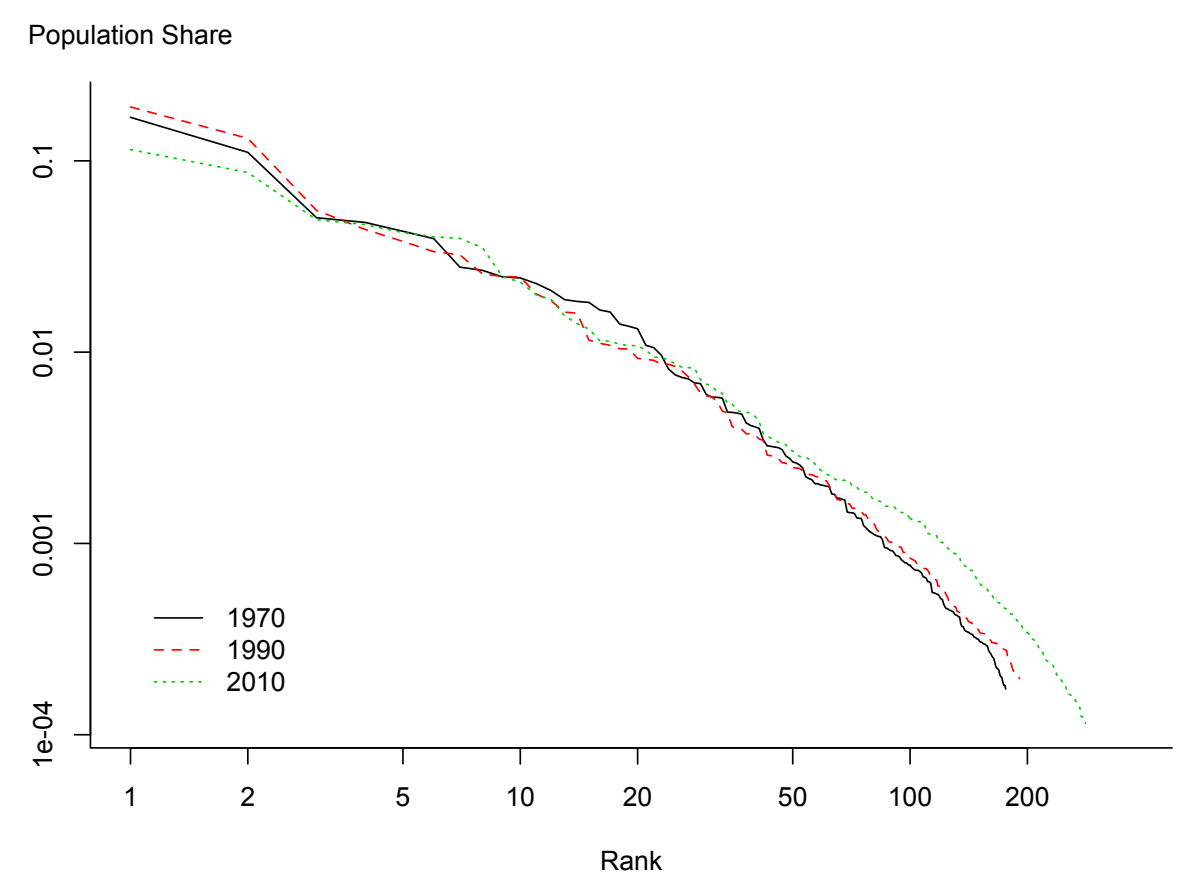

Figure 8: U.S. Hispanic city size distribution using all cities with populations equal to at least $0.1 \%$ of the largest city population, 1970-2010.

\begin{tabular}{|c||c|c|c|c|c|}
\hline & All & White & Black & Hispanic & Asian \\
\hline 1910 & 6.46 & 10.37 & 151.95 & & \\
1950 & 2.23 & 2.32 & 64.92 & & \\
1960 & 5.09 & 5.72 & 48.53 & & \\
1970 & 14.56 & 12.92 & 43.24 & 19.65 & \\
1980 & 4.27 & 5.32 & 23.52 & 11.57 & 3.34 \\
1990 & 4.63 & 5.69 & 26.34 & 12.45 & 2.40 \\
2000 & 4.58 & 6.01 & 31.63 & 12.21 & 4.79 \\
2010 & 6.29 & 8.57 & 36.18 & 21.69 & 6.33 \\
\hline
\end{tabular}

Table 3: Mean squared deviation (\%) from estimated power law distribution for U.S. white, Black, Hispanic, and Asian populations using all cities with populations equal to at least $0.1 \%$ of the largest city population, 1910-2010. 


\begin{tabular}{|c||c|c|c|c|c|}
\hline & All & White & Black & Hispanic & Asian \\
\hline 1910 & 160 & 160 & 153 & & \\
1950 & 160 & 160 & 145 & & \\
1960 & 203 & 203 & 182 & & \\
1970 & 275 & 275 & 225 & 176 & \\
1980 & 331 & 330 & 251 & 198 & 228 \\
1990 & 366 & 365 & 274 & 191 & 230 \\
2000 & 366 & 365 & 294 & 231 & 261 \\
2010 & 371 & 364 & 315 & 282 & 281 \\
\hline
\end{tabular}

Table 4: Number of cities with populations greater than or equal to $0.1 \%$ of largest city population for U.S. white, Black, Hispanic, and Asian populations, 1910-2010. 